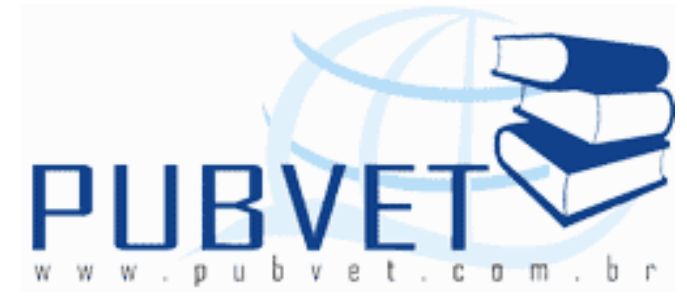

PUBVET, Publicações em Medicina Veterinária e Zootecnia.

Disponível em: <https://doi.org/10.31533/pubvet.v02n11a441>.

\title{
Aplicação da técnica da taxa de depuração renal de creatinina para avaliação do "status" do fósforo em eqüinos"
}

Paulo Reis de Carvalho², Raul Dantas d'Arce3, Raul Machado Neto³, Airton Manzano ${ }^{4}$

${ }^{1}$ Apoio financeiro da EMBRAPA - UEPAE, São Carlos-SP.

2 Parte da Dissertação de mestrado do primeiro autor.

2 Méd. Vet., Dr., Pesquisador, APTA Regional - Secretaria Agricultura e Abastecimento, Bauru-SP. E-mail: p_reiscar@apta.sp.gov.br

3 Professor da Escola Superior de Agricultura Luiz de Queiroz -ESALQ/USP, Piracicaba - SP.

${ }^{4}$ Engo Agro., Dr., Pesquisador, EMBRAPA-UEPAE, São Carlos-SP.

Resumo - Objetivou-se avaliar a eficiência da determinação da taxa do clearance de creatinina para o fósforo ( $\%$ DRCr- P) como ferramenta de diagnóstico na prevenção da osteopatias em eqüinos recebendo rações contendo diferentes proporções de cálcio e de fósforo nas dietas associadas a coletas diárias de sangue e urina nos tempos de 0, 48, 72, 96 e 120 horas após período de adaptação de 15 dias. Foram utilizadas 20 potras árabe e mestiça árabe com idade média de 19,9 \pm 4,58 meses, em delineamento inteiramente casualizado, com cinco potras por tratamento, submetidas a 
quatro proporções de cálcio e de fósforo na dieta por dia: A- Cálcio ( $\mathrm{Ca}$ ) normal e $\mathrm{P}$ normal: $24 \mathrm{~g}$ de $\mathrm{Ca}$ e $18 \mathrm{~g}$ de $\mathrm{P}$; $\mathrm{B}$ - Ca baixo e $\mathrm{P}$ alto: 4,42 $\mathrm{g}$ de $\mathrm{Ca}$ e 30,10 g de P; C - Ca baixo e P baixo: 15,6 g de Ca e 9,6 g de P; e D - Ca alto e $P$ baixo: $29,04 \mathrm{~g}$ de $\mathrm{Ca}$ e $14,04 \mathrm{~g}$ de $\mathrm{P}$.

As médias variaram $(P>0,05)$ de $0,83 \%(0 \mathrm{~h})$ a $2,52 \%(96 \mathrm{~h})$ para o $\% \mathrm{DRCr}-\mathrm{P}$ no tratamento $A$. Proporcionalmente ao decréscimo de $P$ na dieta, as médias para o \%DRCr- $P$ também decresceram $(P>0,05)$ de $1,41 \%$ a $0,62 \%$ no grupo $C$ e de $1,25 \%$ a $0,79 \%$ no grupo $D$. O contrario foi assinalado com o acréscimo de $P$ em excesso no tratamento $B$ em que as médias apresentaram acréscimos $(\mathrm{P}<0,05)$ de $2,10 \%(0 \mathrm{~h})$ e de $5,92 \%(48 \mathrm{~h})$ a $22,71 \%(120 \mathrm{~h})$, sendo os contrastes destas médias significativos entre tempos e tratamentos. As excreções médias de $P$ aumentaram $(P<0,05)$ de 4,15 mg $(0 \mathrm{~h})$ para $19,91 \mathrm{mg}$ (48 h) a 31,20 mg P/dL ( 96 h) no grupo $B$ (alto $P$ ) e opostamente decresceram nos tempos $(120 \mathrm{~h})$ dos grupos $C(3,06 \mathrm{mg} P)$ e $D(1,20 \mathrm{mg} P)$ com $P$ deficiente na dieta. Estes resultados permitiram concluir que a técnica do \% DRCr- $P$ detectou com precisão em tempo hábil (48 h) se a dieta tinha excessivo $\mathrm{P}$ de acordo com a variação de médias obtidas nos grupos A e B.

Palavras-chave: taxa clearance creatinina, osteodistrofia, urina, potra árabe

\section{Application of technique of the renal purification of creatinine ratio for evaluation of phosphorus status in equine}

Abstract - This experiment was carried out to evaluate the efficiency of creatinine clearance ratios for the phosphorus (\% DRCr-P) as a diagnostic tool for the prevention of osteopathies in horses receiving rations containing different proportions of calcium ( $\mathrm{Ca}$ ) and phosphorus ( $\mathrm{P}$ ) in the rations associated with collections daily blood and urine in times of 0,48, 72, 96 and 120 hours after adjustment period of 15 days. Twenty fillies means $19,9 \pm$ 4,58 months of age Arabian and crossbred Arabian were used, in a randomized 
design, with five fillies for treatment, submitted the four proportions of $\mathrm{Ca}$ and $\mathrm{P}$ in the diet for day: normal calcium ( $\mathrm{Ca}$ ) and normal P: $24 \mathrm{~g}$ of $\mathrm{Ca}$ and $18 \mathrm{~g}$ of P; B - Ca low and high P: 4,42 g of Ca and 30,10 g of P; C - low Ca and low P: 15,6 $\mathrm{g}$ of $\mathrm{Ca}$ and 9,6 $\mathrm{g}$ of $\mathrm{P}$; and $\mathrm{D}-$ high $\mathrm{Ca}$ and low P: 29,04 $\mathrm{g}$ of $\mathrm{Ca}$ and $14,04 \mathrm{~g}$ of $\mathrm{P} /$ day in the diet. Proportionately to $\mathrm{P}$ decrease in the diet, means for the \%DRCr- $P$ also decreased $(P>0.05)$ from $1.41 \%$ to $0.62 \%$ in the $C$ group and from $1.25 \%$ to $0.79 \%$ in the $D$ group. On the other hand, we found that with addition of $P$ overpaid in the $B$ treatment means increased $(P<0.05)$ from $2.10 \%(0 \mathrm{~h})$ and from $5.92 \%(48 \mathrm{~h})$ to $22.71 \%(120 \mathrm{~h})$, where these means contrasts were significant on time and treatments. $P$ average excretions increased $(P<0.05)$ of $4.15 \mathrm{mg}(0 \mathrm{~h})$ for $19.91 \mathrm{mg}(48 \mathrm{~h})$ to $P 31.20 \mathrm{mg} / \mathrm{dL}$ (96 h) in B group (high P) and in contrary decreased for times (120 hours) of $C(3.06 \mathrm{mg} \mathrm{P} / \mathrm{dL})$ and $D(1.20 \mathrm{mg} P / \mathrm{dL})$ groups with $P$ deficient in the diet. These results showed that \%DRCr-P technique detect accurately in a skilful time (48 $\mathrm{h}$ ) if the diet has excess of $\mathrm{P}$ according to means variation obtained in groups A and B.

Key Words: creatinine clearance ratio, osteodystrophy, urine, arabian fillie

\section{Introdução}

O rim desempenha papel diferenciado na homeostasia do cálcio (Ca) e P na espécie eqüina. Schryver et al. (1970) e Whitlock et al. (1971) afirmaram que a digestibilidade real do $\mathrm{P}$ apresentou média $45 \%$ quando administrado em nível de dieta de mantença. Entretanto, a absorção do $\mathrm{P}$ da dieta pode variar $30 \%$ a $50 \%$ e a perda fecal endógena tem apresentado média de $10 \mathrm{mg} / \mathrm{kg}$ PV/dia (Schryver et al., 1971b; Cymbaluk \& Christensen, 1986) e é relativamente constante sobre ampla variação de ingestão destes minerais (Schryver et al., 1974). Ao contrário dos ruminantes, a excreção urinária de $P$ 
é sempre significativa em cavalos e é afetada pela dieta (Schryver et al., 1971b).

Segundo Schryver et al. (1971b, 1974), a excreção endógena renal aumentou de $1,5 \mathrm{mg}(0,20 \% \mathrm{P}$ - exigência de mantença) para $42 \mathrm{mg} / \mathrm{kg}$ $\mathrm{PV} /$ dia com $\mathrm{P}$ alto $(1,19 \% \mathrm{P})$ na dieta, evidenciando linearidade crescente da excreção de $\mathrm{P}$ na urina em função do aumento do consumo (Cymbaluk \& Christensen, 1986). Portanto, se eqüinos com alto $P$ na dieta absorvem e excretam maior teor de fosfatos $\left(\mathrm{PO}_{4}{ }_{4}\right)$ na urina, a determinação da taxa do clearance de creatinina (Cr) para $\mathrm{PO}_{4}$ (\% DRCr-P) segundo Traver et al. (1976), Coffman (1980), Caple et al. (1982a, b), Mason et al. (1988), é considerada ferramenta estratégica na detecção precoce do desequilíbrio iônico do $\mathrm{P}$ ao permitir diagnosticar e corrigir o problema antes que ocorram lesões irreversíveis ao tecido esquelético (Knight et al. 1985).

Por outro lado, a prática do calculo do \%DRCr- $\mathrm{P}$ é considerada aplicável em situações específicas onde exista quadros subclínicos e clínicos compatíveis com disfunção do mineral, determinantes de osteopatias agudas ou crônicas, entre outras, osteodistrofia fibrosa, osteítes, deformidades angulares, contratura dos tendões flexores, osteocondrose, laminites, fraturas de vértebras lombares e sacras (Hintz, 1979; Mason et al. 1988). Nestes, além da análise bromatológica completa dos alimentos, as análises bioquímicas do P no soro e urina podem ser realizadas, em face da complexidade das inter-relações minerais, do metabolismo animal e de amplas variações na digestibilidade real do $\mathrm{P}$ dos diferentes ingredientes da dieta. Desta maneira, esta pesquisa teve como objetivo avaliar a eficiência do \% DRCr- $\mathrm{P}$ em detectar precocemente através de dosagens bioquímicas no soro e urina amostrados simultaneamente nos diferentes tempos de coleta, 0 status nutricional do $\mathrm{P}$ em eqüinos submetidos a dietas com teores variáveis de $\mathrm{P}$ em condições experimentais controladas. 


\section{Material e Métodos}

A fase experimental do presente projeto de pesquisa foi desenvolvida na Unidade de Pesquisa de Âmbito Estadual da EMBRAPA de São Carlos-SP, clima tipo Cw, subtropical chuvoso, inverno seco e precipitação anual média de $1502 \mathrm{~mm}$.

Foram utilizadas 20 potras, sendo 10 potras puras da raça Árabe e 10 mestiças Árabe, com idade e desvio padrão médios de 19,9 $\pm 4,58$ meses. 0 peso e altura médios das potras e o desvio padrão no início do experimento foram, respectivamente, de $277,2 \mathrm{~kg} \pm 37,76$ e $1,37 \mathrm{~m} \pm 0,05$. O peso e altura médios das potras e o desvio padrão no final do experimento foram, respectivamente, de $284,8 \mathrm{~kg} \pm 33,95$ e 1,40 $\mathrm{m} \pm 0,04$.

Os animais foram distribuídos em baias individuais de alvenaria com área de $11,97 \mathrm{~m}^{2}(4,20 \mathrm{~m} \times 2,85 \mathrm{~m})$, piso de cimento, providas de cocho de cimento onde recebiam as rações completas (volumoso mais concentrado) nos tratamentos especificados. Durante as fases de adaptação e experimental não foram usados qualquer tipo de cama sobre o piso.

Os tratamentos em número de quatro, foram aplicados a grupos de cinco potras e consistiam das seguintes proporções de $\mathrm{Ca}$ e $\mathrm{P}$ por dia na dieta: A- Ca normal e P normal: $24 \mathrm{~g}$ de Ca e $18 \mathrm{~g}$ de $\mathrm{P}$; $\mathrm{B}$ - Ca baixo e $\mathrm{P}$ alto: 4,42 $\mathrm{g}$ de $\mathrm{Ca}$ e 30,10 g de P; C - Ca baixo e P baixo: 15,6 g de Ca e 9,6 g de P; e D - Ca alto e P baixo: 29,04 g de Ca e 14,04 g de P por dia na dieta de acordo com a Tabela 1, associadas a diferentes tempos de coleta, após o período de adaptação dos animais. Durante o período experimental, os animais receberam $6 \mathrm{~kg}$ de matéria seca ( $2 \%$ do peso vivo) a partir de formulações completas por dia em duas refeições, sendo a primeira as $10 \mathrm{~h}$ e a segunda as $16 \mathrm{~h}$. A composição química bromatológica dos ingredientes das dietas experimentais foi determinada segundo a metodologia descrita pelo AOAC (1970). A composição mineral em $\mathrm{Ca}$ e $\mathrm{P}$ das dietas experimentais foram determinadas em espectrofotômetro de absorção atômica da marca Varian ${ }^{\circledR}$. 
O sangue total para obtenção do soro foi colhido por punção da jugular em tubo de vidro tipo vacutainer sem anticoagulante. A urina foi colhida diretamente da bexiga com auxílio de sonda metálica de inox esterelizada e precedida de cuidadosa assepsia do local. Após 15 dias de adaptação, as amostras de urina e soro foram obtidas diariamente com colheita simultânea no horário entre $12 \mathrm{~h}$ e $14 \mathrm{~h}$ nos tempos 0 (inicial), 48, 72, 96 e $120 \mathrm{~h}$ do início da fase experimental. O P e $\mathrm{Cr}$ no soro e urina para determinação do clearance percentual de $\mathrm{P}(\% \mathrm{DRCr}-\mathrm{P})$ foram dosados pelo método kit labtest Sistemas Diagnósticos Ltda., por reação colorimétrica com leitura em espectrofotômetro modelo 382-B. O princípio do método se baseia em: o íon fosfato reage com molibdênio em meio ácido, tornando-se amarelo, com leitura em $650 \mathrm{~nm}$. A creatinina reage com solução de picrato em alcalino, formando um complexo de cor vermelha com leitura em $510 \mathrm{~nm}$. Foi aplicada a formula estabelecida por Knudsen (1959) e adaptada por Travers et al. $(1976,1977)$ na qual os autores interrelacionaram a concentração do mineral pesquisado $(P)$ na urina e soro com a concentração de creatinina $(\mathrm{Cr})$ no soro e urina de eqüinos para obter o porcentual de depuração renal (\%DRCr-) do mineral pesquisado:

\begin{tabular}{|c|c|c|}
\hline \multirow{3}{*}{$\%$ DRCr- $\mathrm{P}=$} & $P$ (urina) & $\mathrm{Cr}$ (soro) \\
\hline & ------- $\quad x$ & --- x 100 \\
\hline & $\mathrm{P}$ (soro) & $\mathrm{Cr}$ (urina) \\
\hline
\end{tabular}

$\mathrm{Na}$ análise estatística dos dados, cada tratamento foi aplicado a cada unidade inteiramente ao acaso, a cinco animais. Os resultados das determinações do \%DRCr- $\mathrm{P}, \mathrm{Cr}$ no soro e urina foram analisados nos tempos de $0,48,72,96$ e $120 \mathrm{~h}$ para cada unidade experimental. Foram determinados os contrastes e interações de médias entre tempos e tratamentos. Para avaliar o comportamento das variáveis no $\mathrm{P}$ e $\mathrm{Cr}$ no soro e urina e \% $\mathrm{DRCr}-\mathrm{P}$ médio nos tempos também foram realizadas a análise conjunta dos tempos nos respectivos tratamentos. Os dados foram processados mediante o uso do software "Statistical Analysis System" (SAS, 1994). O efeito dos tempos foi 
Carvalho, P.R., d'Arce, R.D., Machado Neto, R. et al. Aplicação da técnica da taxa de depuração renal de creatinina para avaliação do "status" do fósforo em eqüinos. PUBVET, Londrina, V. 2, N. 45, Art\#441, Nov2, 2008.

estudado através da regressão polinomial, enquanto que as médias dos tratamentos foram comparadas pelo teste de Tukey ao nível de significância indicado.

Tabela 1 - Composição das rações experimentais

\begin{tabular}{lcccc}
\hline Ingredientes (\%) & \multicolumn{4}{c}{ Tratamentos } \\
\cline { 2 - 5 } & $\mathrm{A}$ & $\mathrm{B}$ & $\mathrm{C}$ & $\mathrm{D}$ \\
\hline Feno de Coast cross & 59,2245 & 16,6550 & 100,0000 & 59,2660 \\
Farelo de milho & 26,7000 & 49,4600 & - & 26,700 \\
Farelo de trigo & 4,0000 & 33,3400 & - & 4,0000 \\
Farelo de soja & 9,3000 & - & - & 9,3000 \\
Carbonato de cálcio (p.a) & 0,5235 & - & - & 0,7340 \\
Fosfato monosódico (p.a) & 0,2520 & 0,5450 & - & - \\
\hline Total & 100,0000 & 100,0000 & 100,0000 & 100,0000 \\
\hline Análise calculada (\%) & & & & \\
Matéria seca & 88,7074 & 88,0207 & 89,3700 & 88,6818 \\
Proteína bruta & 10,0158 & 10,1738 & 4,6300 & 10,0180 \\
Fibra bruta & 25,0423 & 10,1505 & 39,5200 & 25,0609 \\
Extrato etéreo & 2,8838 & 3,1448 & 2,5000 & 2,8850 \\
Matéria mineral & 3,9734 & 2,6494 & 4,9900 & 3,9758 \\
Extrativo não nitrogenado & 45,8684 & 61,3726 & 37,7300 & 45,8861 \\
\hline Composição analisada (\%) & & & & \\
Cálcio & 0,4000 & 0,0736 & 0,2600 & 0,4840 \\
Fósforo & 0,3000 & 0,5016 & 0,1600 & 0,2338 \\
\hline
\end{tabular}




\section{Resultados e Discussão}

O efeito dos tratamentos, dos tempos e interações entre tempos e tratamentos são apresentados na tabela 2 abaixo.

Foi observado efeito não significativo $(P>0,05)$ dos tempos do tratamento $A$ para o \%DRCr- $\mathrm{P}$ expresso pela equação de regressão polinomial cúbica $\mathrm{Y}=$ $0,0000005 X^{3}+0,001008 X^{2}-0,038413 X+0,8511$ (Tabelas 3 e 4; Figura 1). Com níveis de ingestão de $18 \mathrm{~g}$ de $\mathrm{P} /$ dia no grupo de $\mathrm{A}(0,30 \%$ de $\mathrm{P} / \mathrm{kg}$ de MS), as potras mostraram variação de médias não significativas $(P>0,05)$ entre tempos de $0,83 \%(0 \mathrm{~h})$ a 2,52\%, maior média e, entre tratamentos no tempo inicial $(0 \mathrm{~h})$ de $0,83 \%$ (A) a $2,10 \%$ (B) para o \% DRCr- P. Nestes tratamentos todos os animais provinham de dietas com níveis de ingestão de $18 \mathrm{~g}$ de P/dia (Tabelas 1 e 4; Figura 1). Segundo Schryver et al. (1972b) a técnica do óxido de crômio $\left(\mathrm{Cr}_{2} \mathrm{O}_{3}\right)$ indicou a existência de fluxo liquido $\mathrm{P}$ na primeira metade e equivalente absorção liquida do elemento na segunda metade do intestino delgado. Segundo os autores, a técnica revelou grande fluxo de $\mathrm{P}$ dentro do ceco e colon ventral e absorção liquida de quantidade maior do mineral no colon dorsal maior e pequeno colon. O conteúdo de $\mathrm{Ca}$ ou tipo de alimento não alteraram o sítio de secreção ou a absorção de $\mathrm{P}$ do eqüino, diferentemente dos ovinos, suínos e cão nos quais existe pouca absorção de $\mathrm{P}$ no intestino grosso. Assim, de acordo com os autores acima, estas particularidades no metabolismo do $\mathrm{P}$ caracterizam os rins como 0 principal órgão na regulação da homeostasia do $\mathrm{P}$ em eqüinos traduzindo a importância da técnica do \%DRCr- $\mathrm{P}$, uma vez que a homeostasia do $\mathrm{P}$ no cavalo em primeira instancia é regulada pelos rins, excretando o excesso ou reabsorvendo $P$ do filtrado na deficiência. Para repor continua remoção óssea de $\mathrm{P}$ imposta pelo mecanismo de turnover, há necessidade de absorção de quantidade equivalente do mineral. Nesta pesquisa foi calculada a ingestão total de $6 \mathrm{~kg}$ de MS ( $2 \%$ do peso vivo) para potras em crescimento entre $18 \mathrm{a}$ 24 meses de idade com base no atendimento de $100 \%$ (0,30\% P: $18 \mathrm{~g} \mathrm{P} /$ dia) 
Carvalho, P.R., d'Arce, R.D., Machado Neto, R. et al. Aplicação da técnica da taxa de depuração renal de creatinina para avaliação do "status" do fósforo em eqüinos. PUBVET, Londrina, V. 2, N. 45, Art\#441, Nov2, 2008.

da exigência do NRC (1978), 136,37\% (0,22\% P:13,2 g/dia) do NRC (1989) e 115,38\% (0,26\% P: 15,6 g/dia) do NRC (2007) (Tabela 1; Figura 1).

Tabela 2 - Análise de variância para efeito dos tratamentos, tempos e interação entre tempo $x$ tratamento

\begin{tabular}{lccccc}
\hline Fonte de variação & G.L & $\begin{array}{c}\text { Soma dos } \\
\text { quadrados }\end{array}$ & $\begin{array}{c}\text { Quadrado } \\
\text { médio }\end{array}$ & $\begin{array}{c}\text { Valor de } \\
\mathrm{F}\end{array}$ & $\begin{array}{c}\text { Probabi- } \\
\text { lidade>F }\end{array}$ \\
\hline Modelo & 19 & $2.717,98$ & 143,05 & 7,33 & 0,0001 \\
Resíduo & 80 & $1.560,80$ & 19,51 & & \\
Total & 99 & $4.278,77$ & & & \\
\hline Fonte de variação & G.L & Erro tipo I & Quadrado & Valor de & Pr>F \\
& & & médio & $\mathrm{F}$ & \\
\hline Tempo (T) & 4 & 326,05 & 81,51 & 4,18 & 0,0040 \\
Tratamento (Trata) & 3 & $1.391,03$ & 463,68 & 23,77 & 0,0001 \\
Tx Trata & 12 & $1.000,89$ & 83,41 & 4,28 & 0,0001 \\
\hline Fonte de variação & G.L & Erro tipo III & Quadrado & Valor de & Pr>F \\
& & & médio & $\mathrm{F}$ & \\
\hline Tempo & 4 & 326,05 & 81,51 & 4,18 & 0,0040 \\
Tratamento & 3 & $1.391,03$ & 463,68 & 23,77 & 0,0001 \\
Tx Trata & 12 & $1.000,89$ & 83,41 & 4,28 & 0,0001 \\
\hline
\end{tabular}


Tabela 3 - Equações de regressão polinomial cúbica ajustadas e coeficientes de determinação do total de $\mathrm{P}$ ingerido ( $\mathrm{g} / \mathrm{dia}$ ) nas dietas segundo os tratamentos estudados

\begin{tabular}{|c|c|c|c|}
\hline $\begin{array}{l}\text { Tratamento/ } \\
\text { P (g/dia) na } \\
\text { dieta }\end{array}$ & Equações ajustadas & $\mathrm{R}^{2}$ & $\mathrm{P}^{*}$ \\
\hline$A-18 g$ & $\begin{array}{l}Y=-0,0000005 X^{3}+0,001008 X^{2}-0,038413 X+ \\
0,8511\end{array}$ & 0,8740 & n.s \\
\hline $\mathrm{B}-30,10 \mathrm{~g}$ & $Y=0,0001 X^{3}-0,0102 X^{2}+0,4838 X+1,8405$ & 0,8095 & $<0,05$ \\
\hline $\mathrm{C}-9,6 \mathrm{~g}$ & $\begin{array}{l}Y=-0,0000002 X^{3}-0,0000155 X^{2}-0,00202 X+ \\
1,3216\end{array}$ & 0,7591 & n.s \\
\hline$D-14,04 \mathrm{~g}$ & $\begin{array}{l}Y=-0,000003 X^{3}+0,000433 X^{2}-0,019428 X+ \\
1,2560\end{array}$ & 0,9293 & n.s \\
\hline
\end{tabular}

* - probabilidade para efeito de tempo

Os animais submetidos à dieta do tratamento A mostraram resultados concordantes aos obtidos nos estudos de balanço $P$ realizados por Schryver et al. (1971b) e revisados por Schryver \& Hintz (1972a) demonstrando que a retenção é zero para a ingestão de $19 \mathrm{mg} P / \mathrm{kg} P V /$ dia. A excreção renal de $P$ foi diretamente proporcional à ingestão. Segundo os autores acima, pôneis apresentaram balanço positivo de $\mathrm{P}$ quando alimentados com dietas contendo porcentuais variáveis de $0,20 \% ; 0,54 \% ; 0,79 \%$ e $1,19 \%$ de $P$, entretanto, retinham mais $\mathrm{P}$ para altos níveis de ingestão. Os autores acima mencionaram ainda que, a regressão linear da excreção urinária sobre a ingestão: $Y=$ $0,240 \mathrm{X}-7,103$, preditou que a excreção foi igual a zero quando a ingestão foi menor que $29 \mathrm{mg} \mathrm{P/kg/PV/dia,} \mathrm{embora} \mathrm{a} \mathrm{análise} \mathrm{de} \mathrm{regressão} \mathrm{linear} \mathrm{possa} \mathrm{não}$ ter representado a descrição mais adequada desta relação para baixos níveis de ingestão. Na presente pesquisa a equação de regressão linear $Y=0,9499 X$ - 9,4037 ( $\left.R^{2}=0,89, R=0,94\right)$ estimou que a excreção renal decresceu, 
tendendo a zero quando a ingestão foi menor que $31,35 \mathrm{mg} \mathrm{P} / \mathrm{kg} \mathrm{PV} /$ dia para potras com média $300 \mathrm{~kg}$ PV. Estes resultados demonstraram ainda que os tratamentos que forneceram 9,6 g P/dia (C) e 14,04 g P/dia (D), a excreção renal mostrou decréscimo não significativo $(P>0,05)$ nos tempos e o inverso ocorreu $(P<0,05)$ com a excreção urinária de $P$ dos animais do grupo $B$ submetidos à dieta com teor elevado de P (Tabela 5; Figura 2).

Entretanto, deve-se acrescentar que com base na variação $30 \%$ a 50\% (média de $40 \%$ a $45 \%$ ) da digestibilidade real do $\mathrm{P}$ obtida nos estudos realizados por Schryver \& Hintz (1972a), estimando exigência de mantença entre 20 a 40 $\mathrm{mg} / \mathrm{kg} \mathrm{PV/dia,} \mathrm{pode} \mathrm{resultar} \mathrm{em} \mathrm{variação} \mathrm{ligeiramente} \mathrm{superior} \mathrm{ou} \mathrm{inferior} \mathrm{que}$ os valores obtidos pela análise de regressão dos estudos de balanço. Por outro lado, estudo com ${ }^{32} \mathrm{P}$ mostrou que a excreção fecal endógena de $\mathrm{P}$ média de 10 $\mathrm{mg} / \mathrm{kg}$ PV/dia não foi influenciada pela ingestão de teores crescentes de 0,20\% $\mathrm{P}$ a $1,20 \% \mathrm{P}$ na ração (Schryver et al., 1974), contrastando com ratos e ruminantes em que a excreção endógena fecal é diretamente proporcional a ingestão, fato que caracterizou os rins como o principal órgão envolvido na homeostasia do $\mathrm{P}$ em eqüinos. Concordando ainda com os resultados da presente pesquisa, Schryver et al.(1972a) encontraram para níveis de ingestão de $\mathrm{P}$ variando de baixo a alto, excreções urinárias de $\mathrm{P}$ proporcionalmente crescentes de P de $1 \mathrm{mg}(0,20 \% \mathrm{P}$ - dieta basal), $11 \mathrm{mg}(0,54 \% \mathrm{P}-\mathrm{P}$ fítico), $19 \mathrm{mg}(0,79 \% \mathrm{P})$ e $42 \mathrm{mg} / \mathrm{kg} \mathrm{PV} /$ dia $(1,19 \%)$ de $\mathrm{P}$ na urina segundo os teores aumentados de $\mathrm{P}$ na dieta. Entretanto, os resultados desta pesquisa e dos experimentos citados na presente literatura discordam de Lopes et al. (2003) ao relatarem resultado de pesquisa com efeito $(P>0,05)$ não significativo da quantidade de $\mathrm{P}$ excretada na urina em função dos níveis de $\mathrm{P}$ consumido nas dietas. Se a média de consumo de $P$ é abaixo das exigências, o $\% \mathrm{DRCr}-\mathrm{P}$ também apresenta valores reduzidos. Portanto, nesta pesquisa, os resultados baseados em médias de consumo diário de $P$ também são concordantes com as médias obtidas por Caple et al. (1982a) ao mencionarem que em oito éguas adultas de 3 a 6 anos de idade, recebendo dieta com 0,20\% de P, apresentaram \% DRCr- $\mathrm{P}$ menor que $4 \%$, o que representou no estudo de 
excreção de $P$ na urina valor menor que $4,65 \mathrm{mg} / \mathrm{dL}$. Entretanto, Joyce et al. (1971) avaliaram nove eqüinos normais e obtiveram valores variando de 4 a $18 \mathrm{mg} / \mathrm{dL}$ de $\mathrm{P}$ na urina em dietas equilibradas em Ca e P. Concordando com os autores acima, os resultados desta pesquisa expressaram variação de médias de 2,27 mg $(A-0 h)$ a 4,15 mg/dL $(B-0 h)$ entre tratamentos e de $1,38 \mathrm{mg}(48 \mathrm{~h})$ a $7,55 \mathrm{mg} / \mathrm{dL}(96 \mathrm{~h})$ de $\mathrm{P}$ na urina entre tempos dos eqüinos submetidos ao tratamento A (Tabela 4). Concordando com Van Doorn et al. (2004) de que tais variações podem ser em parte devido a digestibilidade real do $\mathrm{P}$ envolvendo o $\mathrm{P}$ fítico dos alimentos e a digestibilidade real do $\mathrm{P}$ disponível nos ingredientes utilizados no preparo das diferentes rações. Desta maneira, Hintz \& Schryver $(1972,1976)$ e Hintz et al. (1973) encontraram 43,5\% de digestibilidade real para o fosfato bicálcico, 29,5\% para o farelo de trigo e dieta basal constituída de feno de timóteo (Phleum pratense) e 39,3\% digestibilidade real para polpa de beterraba, amido e milho. Traver et al. (1976) reportaram médias para o \% DRCr- $\mathrm{P}$ em cavalos adultos clinicamente normais variando de $0 \%$ a $0,5 \%$. A técnica utilizada pelos autores foi baseada em trabalhos realizados por Knudsen (1959), Rawlings et al. (1975) e Zatsman et al.(1985) por meio da determinação do índice de depuração renal de creatinina para o $\mathrm{P}(\% \mathrm{DRCr}-\mathrm{P})$ e foi validada pelos autores como prática diagnóstica. A existência de discrepância dos valores citados na literatura para o \%DRCr- $P$ certamente estão ligadas a não padronização das dietas empregadas nas avaliações, ou seja, quantificação dos teores de $\mathrm{P}$ na dieta final. 
Carvalho, P.R., d'Arce, R.D., Machado Neto, R. et al. Aplicação da técnica da taxa de depuração renal de creatinina para avaliação do "status" do fósforo em eqüinos. PUBVET, Londrina, V. 2, N. 45, Art\#441, Nov2, 2008.

Tabela 4 - Valores médios dos \% DRCr- $\mathrm{P}$ e de $\mathrm{P}$ na urina $(\mathrm{mg} / \mathrm{dL})$ nos tempos $0,48,72,96$ e 120 dos tratamentos estudados

\begin{tabular}{|c|c|c|c|c|c|}
\hline \multirow{4}{*}{ Tratamentos } & \multicolumn{5}{|c|}{ Tempos (h) } \\
\hline & 0 & 48 & 72 & 96 & 120 \\
\hline & \multicolumn{5}{|c|}{ Médias* \% DRCr-P } \\
\hline & \multicolumn{5}{|c|}{ 5\% de significância } \\
\hline$A$ & $0,83^{A}{ }^{A}$ & $1,01_{a}{ }^{B}$ & $1,09 a^{B}$ & $2,52 a^{B}$ & $2,37 a^{B}$ \\
\hline B & $2,10 c^{A}$ & $5,92 b^{A}$ & $12,46 a b^{A}$ & $6,02 b^{A}$ & $22,71_{a^{A}}$ \\
\hline C & $1,41_{a}{ }^{A}$ & $1,39 a^{B}$ & $0,89_{a}^{B}$ & $1,12_{a} \mathrm{~B}$ & $0,62 a^{B}$ \\
\hline D & $1,25 a^{A}$ & $1,00^{B}$ & $1,22 a^{B}$ & $1,08 a^{B}$ & $0,79_{a} B$ \\
\hline
\end{tabular}

$1 \%$ de significância

\begin{tabular}{lllclc}
$\mathrm{A}$ & $0,83 \mathrm{a}^{\mathrm{A}}$ & $1,09 \mathrm{a}^{\mathrm{B}}$ & $1,10 \mathrm{a}^{\mathrm{B}}$ & $2,52 \mathrm{a}^{\mathrm{B}}$ & $2,37 \mathrm{a}^{\mathrm{B}}$ \\
$\mathrm{B}$ & $2,10 \mathrm{c}^{\mathrm{A}}$ & $5,92 \mathrm{ab}^{\mathrm{A}}$ & $12,46 \mathrm{ab}^{\mathrm{A}}$ & $6,02 \mathrm{ab}^{\mathrm{A}}$ & $22,71 \mathrm{a}^{\mathrm{A}}$ \\
$\mathrm{C}$ & $1,41 \mathrm{a}^{\mathrm{A}}$ & $1,39 \mathrm{a}^{\mathrm{B}}$ & $0,89 \mathrm{a}^{\mathrm{B}}$ & $1,12 \mathrm{a}^{\mathrm{B}}$ & $0,62 \mathrm{a}^{\mathrm{B}}$ \\
$\mathrm{D}$ & $1,25 \mathrm{a}^{\mathrm{A}}$ & $1,00 \mathrm{a}^{\mathrm{B}}$ & $1,22 \mathrm{a}^{\mathrm{B}}$ & $1,08 \mathrm{a}^{\mathrm{B}}$ & $0,79 \mathrm{a}^{\mathrm{B}}$ \\
\hline
\end{tabular}

Médias de $P$ na urina $(\mathrm{mg} / \mathrm{dL}),(P<0,05)$

\begin{tabular}{llcccc}
$\mathrm{A}$ & $2,27 \mathrm{a}^{\mathrm{A}}$ & $1,38 \mathrm{a}^{\mathrm{B}}$ & $4,13 \mathrm{a}^{\mathrm{B}}$ & $7,55 \mathrm{a}^{\mathrm{B}}$ & $5,56 \mathrm{a}^{\mathrm{B}}$ \\
$\mathrm{B}$ & $4,15 \mathrm{~b}^{\mathrm{A}}$ & $19,91 \mathrm{a}^{\mathrm{A}}$ & $25,2 \mathrm{a}^{\mathrm{A}}$ & $31,20 \mathrm{a}^{\mathrm{A}}$ & $24,03 \mathrm{a}^{\mathrm{A}}$ \\
$\mathrm{C}$ & $3,76 \mathrm{a}^{\mathrm{a}}$ & $2,03 \mathrm{a}^{\mathrm{B}}$ & $2,52 \mathrm{a}^{\mathrm{B}}$ & $2,67 \mathrm{a}^{\mathrm{B}}$ & $3,06 \mathrm{a}^{\mathrm{B}}$ \\
$\mathrm{D}$ & $3,49 \mathrm{a}^{\mathrm{A}}$ & $3,09 \mathrm{a}^{\mathrm{B}}$ & $2,68 \mathrm{a}^{\mathrm{B}}$ & $2,65 \mathrm{a}^{\mathrm{B}}$ & $1,20 \mathrm{a}^{\mathrm{B}}$ \\
\hline
\end{tabular}

*Médias com letras distintas minúsculas na linha ou maiúsculas na coluna diferem entre si pelo teste de Tukey ao nível de significância indicado 
Entretanto, os estudos de balanço com ${ }^{32} \mathrm{P}$ realizados por Schryver et al. (1971b) elucidaram o comportamento fisiológico dos eqüinos quanto ao metabolismo do $\mathrm{P}$ permitindo interpretações mais acertadas de tais resultados relatados na literatura. Outros autores apresentaram importantes contribuições quanto ao \% DRCr- P, sendo: que Lane et al. (1983) ao utilizarem quatro cavalos e um pônei, encontraram valor médio de 0,18\%; Konh et al. (1986) encontraram em seis éguas adultas normais valores variando de 0,04\% a $0,16 \%$; Harris e Colles (1988) obtiveram valores variando de 0 a $0,2 \%$; e Edwards et al. (1989) analisaram 65 eqüinos adultos e obtiveram valores variando de $0,023 \%$ a $2,77 \%$ do $\%$ DRCr- P. Com base nas considerações acima e dados de literatura, nesta pesquisa os valores do $\% \mathrm{DRCr}-\mathrm{P}$ encontram-se dentro da margem de variação esperada para variável dependente $P$ na dieta, ou seja, apresentaram valores menores que $4 \%$ citado por Caple et al. (1982a, b) ao variarem de $0,83 \%$ a 2,52\% para o $\% \mathrm{DRCr}-\mathrm{P}$.

Nesta pesquisa, animais recebendo $30,10 \mathrm{~g} \mathrm{P}(0,50 \% \mathrm{P}) /$ dia na ração do tratamento B, com atendimento da exigência de 166,67\% do NRC (1978 0,30\% P), 227,28\% NRC (1989 - 0,22\% P) e 192,31\% NRC (2007-0,26\% P) de $P$ na MS base, foi responsável pelos efeitos $(P<0,05)$ significativos dos contrastes de médias entre os tratamentos $A(0,30 \% \mathrm{P}), \mathrm{C}(0,16 \% \mathrm{P})$ e $\mathrm{D}$ $(0,23 \% \mathrm{P})$ e entre tempos para o \%DRCr- $\mathrm{P}$ (Tabelas 1 e 2$)$. Neste grupo de potras $(B)$ os acréscimos $(P<0,05)$ de médias variaram de $2,10 \%(0 \mathrm{~h}), 5,92 \%$ $(48 \mathrm{~h})$ a $22,71 \%(120 \mathrm{~h})$ entre tempos, representando acréscimo maior que 10 vezes no \%DRCr- P (Tabela 4), certamente determinou aumento da absorção por influência primária do alto $P$ na ração $e$ da regulação homeostatica do PTH sobre os rins via aumento da excreção urinária de P. Tais aumentos do \%DRCr- $\mathrm{P}$ podem ser projetados pela equação de regressão polinomial cúbica: $Y=0,0001 X^{3}-0,0102 X^{2}+0,4838 X+1,8405 ; R^{2}=$ $0,8095, P<0,0352$ e desvio $(P<0,0046)$ significativo entre os tratamentos (Tabela 3; Figura 1). Os resultados das médias deste mesmo grupo de animais (B) submetidos à análise conjunta, apresentaram a maior média de 9,84\% 
contrastando $(P<0,01)$ com as menores médias igual a $1,57 \%(A), 1,08 \%(C)$ e $1,45 \%$ (D) para o \% DRCr- P (Tabela 5; Figura 2).

Na literatura há menção do tempo de 48 horas para detecção de desequilíbrios desta natureza através da determinação do \% DRCr- P (Traver et al., 1976; Coffman, 1980; Caple et al., 1982a, b), sendo usado para monitorar dieta rica em grão e pobre em Ca. Médias do \% DRCr- P maiores que 4\% segundo Caple et al., (1982a, b) e Mason et al. (1988) são indicativas de desequilíbrio na dieta. Nesta pesquisa, a excreção urinária média de $P$ no tratamento $B$ variou de $4,15 \mathrm{mg}(0 \mathrm{~h})$ para $31,20 \mathrm{mg}(96 \mathrm{~h})$ representando acréscimo $(P<0,05)$ entre tempos de 7,5 vezes a maior (Tabela 4; Figura 1). Estes resultados concordam com a afirmação de Schryver et al. (1971a) os quais relataram que teores altos de $\mathrm{P}$ na dieta $(1,20 \% \mathrm{P})$ determinaram aumento na taxa de deposição e remoção do Ca ósseo (osteólise) e aumento da excreção de P na urina influenciada pelo paratormônio que age sobre os rins aumentando a excreção de $\mathrm{PO}_{4}=$ e reduzindo a excreção de Ca. Concordando ainda com as afirmações acima, outros autores teceram importantes considerações com relação ao excesso de $\mathrm{P}$ na dieta. Teeter et al. (1967) e Bourke (1968) examinaram o aparecimento de patologias ósseas em eqüinos, advindas da dieta desequilibrada, com Ca baixo e $\mathrm{P}$ alto. Obtiveram melhora significativa dos animais com a correção dos teores destes elementos na ração; Nordin e Peacock (1969) e Gans \& Mercer (1984) mencionaram o importante papel que os rins exercem sobre a homeostase do $\mathrm{P}$, reabsorvendo do filtrado na deficiência ou excretando quando no excesso do elemento; Argenzio et al. (1974) inferiram que na regulação da homeostase do Ca e $P$, excesso de $P$ e baixo Ca na dieta determinam a hipersecreção da glândula paratireóide com aumento da reabsorção óssea podendo induzir generalizada descalcificação osteodistrófica e aparecimento do Hiperparatiroidismo Nutricional Secundário (HPTNS), quando perdurarem alto teor de $\mathrm{P}$ na dieta associado ao baixo teor de Ca; Mason et al. (1988) analisaram 40 cavalos puro sangue inglês entre três e dez anos de idade, em treino e encontraram valores do \% DRCr-P variando de $0,06 \%$ a $42 \%$. Através da análise da dieta constatou-se excesso 
de $\mathrm{P}$ provenientes da predominância de grãos cereais na ração. Os autores afirmaram que após a correção da dieta, os valores \% DRCr- P normalizaram, sendo menores que $1 \%$; Krook (1968) e Krook \& Lowe (1969) classificaram o HPTNS como sendo de natureza compensatória induzido pela hipocalcemia; Knight et al. (1985) estudaram a incidência das enfermidades ósseas em cavalos e sua correlação com a dieta mineral, enumerando as manifestações clínicas sob a forma de osteocondroses, epifisites, osteoartrites, deformidades angulares dos membros, contratura dos tendões e laminites. Os autores acima observaram correlação igual 0,71 entre os teores dos constituintes da ração total e o aparecimento de problemas ósseos $(P<0,0001)$, ou seja, quanto menor o erro na ração para níveis usados entre $0,50 \%$ a $1,37 \%$ de Ca e $0,38 \%$ a $0,80 \%$ de $\mathrm{P}$, menor o número de problemas ósseos; Schryver et al. (1974) utilizaram dietas altas em $\mathrm{P}(1,4 \% \mathrm{P})$ e observaram que estas dietas promoveram maior taxa de troca de Ca entre o osso e o meio extracelular e maior excreção urinária de $P$.

Tabela 5 - Análise conjunta dos valores médios do \%DRCr- P e médias $(\mathrm{mg} / \mathrm{dL})$ de $\mathrm{P}$ e $\mathrm{Cr}$ no soro e urina de cinco potras medidos nos tempos de $0,48,72,96$ e 120 horas nos tratamentos estudados

\begin{tabular}{|c|c|c|c|c|}
\hline \multirow{3}{*}{ Determinações } & \multicolumn{4}{|c|}{ Tratamentos } \\
\hline & $A$ & $B$ & C & $\mathrm{D}$ \\
\hline & \multicolumn{4}{|c|}{ Médias $^{1}(\mathrm{mg} / \mathrm{dL})$} \\
\hline$\%$ DRCr- $\mathrm{P}$ & $1,57_{b} \mathrm{~B}^{*}$ & $9,84 a^{A}$ & $1,08^{B}$ & $1,45_{b}{ }^{B}$ \\
\hline P no soro & $4,23 a^{A B}$ & $4,77 a^{A}$ & $3,60 b^{B}$ & $4,37 a^{A}$ \\
\hline$P$ na urina & $4,18 b^{B}$ & $20,91_{a^{A}}$ & $2,81_{b}^{B}$ & $2,63 b^{B}$ \\
\hline $\mathrm{Cr}$ no soro & $1,87 b^{A}$ & $1,99 a^{A}{ }^{A}$ & $2,09 a^{A}$ & $1,50 c^{B}$ \\
\hline $\mathrm{Cr}$ na urina & $123,26 b^{A B}$ & $141,62 \mathrm{ab}^{\mathrm{AB}}$ & $207,53 \mathrm{a}^{\mathrm{A}}$ & $97,00_{b} B$ \\
\hline
\end{tabular}

${ }^{1}$ Médias de vinte cinco repetições nos tempos (0, 48, 72, 96 e $\left.120 \mathrm{~h}\right)$ *Médias com letras distintas minúsculas $(P<0,05)$ ou maiúsculas $(P<0,01)$ na linha diferem entre si 


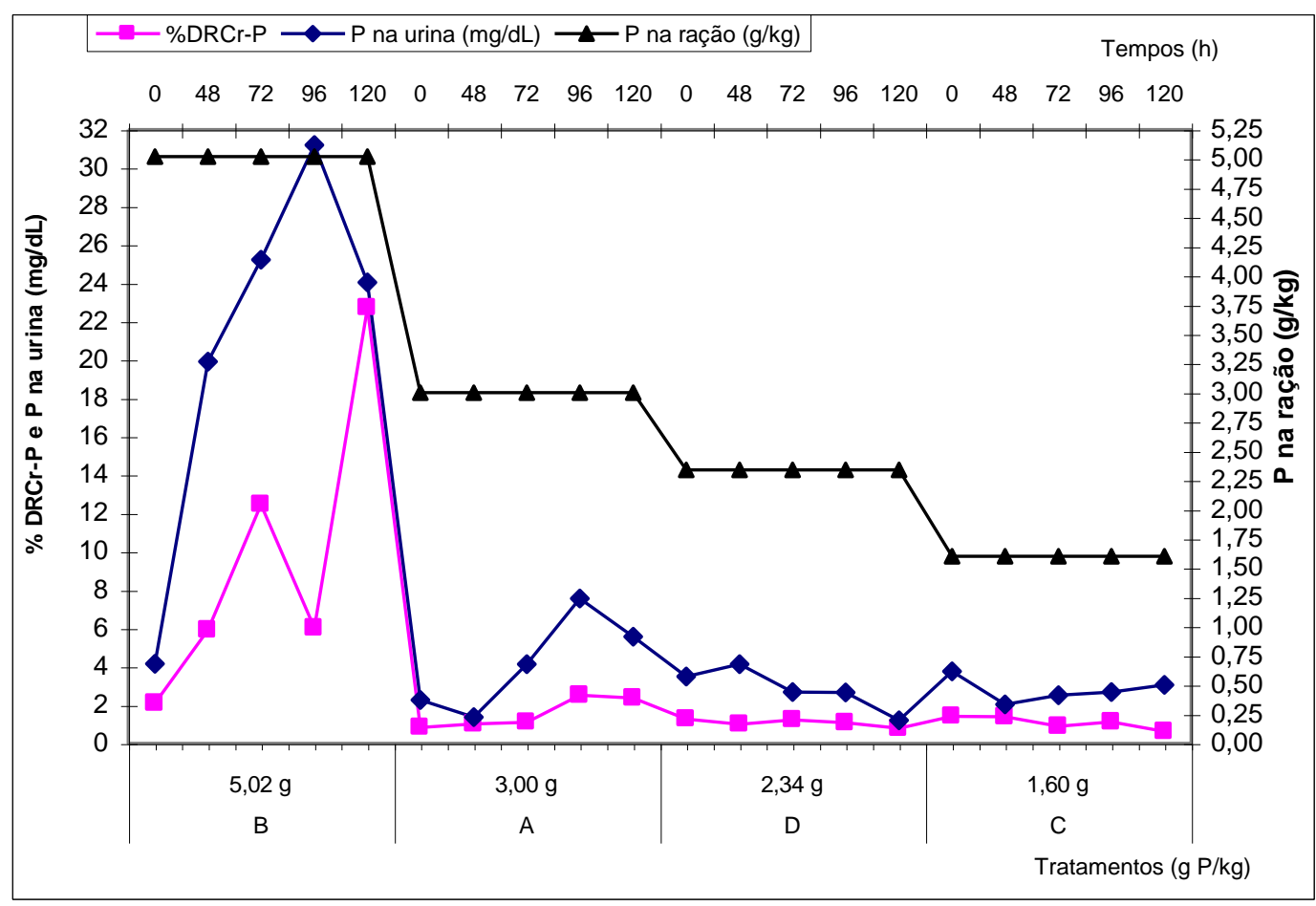

Figura 1- Médias do \% DRCr- $\mathrm{P}$ e de $\mathrm{P}$ na urina $(\mathrm{mg} / \mathrm{dL})$ nos tempos 0, 48, 72, 96 e120 horas em função dos teores de $P$ na ração $(\mathrm{g} / \mathrm{kg})$ segundo os tratamentos estudados

O grupo de potras com 9,6 $\mathrm{g}$ de $\mathrm{P} /$ dia na dieta do tratamento $\mathrm{C}$ (deficiente em $P-0,16 \% P$ ) mostrou decréscimos ( $P>0,05)$ não significativos de $1,41 \%(0 \mathrm{~h})$ a $0,62 \%(120 \mathrm{~h})$, representando redução de $56,03 \%$ no \% DRCr- $\mathrm{P}$, expresso pela equação de regressão polinomial cúbica $\mathrm{Y}=$ $0,0000002 X^{3}-0,0000155 X^{2}-0,00202 X+1,3216$, não significativa entre tempos do tratamento (Tabela 3 ). Neste grupo de animais as exigências foram atendidas em apenas 53,33\% com base no NRC (1978 - 0,30\% P) (Tabela 1), $73,33 \%$ do NRC (1989-0,22\% P) ou em 86,67\% do NRC (2007-0,26\% P) para o consumo total de P na MS base (Tabela 4; Figura 1).

Comportamento semelhante ao dos animais do grupo $\mathrm{C}$ para decréscimo de médias o \% DRCr- P foram apresentados por Cudefford et al. (1990) ao 
descreverem médias de $0,72 \%$ em cavalos adultos normais alimentados com $1,8 \mathrm{~g} \mathrm{P} / \mathrm{kg}$ MS no feno de alfafa (Medicago sativa) e de 0,61\% com 2,3 g P/kg MS no feno de timóteo. Caple et al.(1982a), utilizando cavalos adultos ingerindo dieta sob condições de pastejo e dieta contendo 9,0 g P/dia (relação Ca : P igual 1,2 : 1), obtiveram, respectivamente, médias de $0,08 \%$ e $0,37 \%$ para $0 \%$ DRCr- P, ou seja, menores que 4\%. Caple et al. (1982b) encontraram valores menores que $4 \%$ com dieta equilibrada e maiores que $4 \%$ quando a dieta era alta em $\mathrm{P}(6,1 \mathrm{~g} \mathrm{P} / \mathrm{kg} \mathrm{MS})$, o que também foi verificado no presente experimento na dieta com $P$ alto $(5,02 \mathrm{~g} \mathrm{P} / \mathrm{kg} \mathrm{MS})$ no tratamento $B$.

Nesta pesquisa, o grupo $C$ na análise conjunta dos tempos apresentou valor decrescente de $P$ sérico $(3,60 \mathrm{mg} P / d L$, menor média) diferindo $(P<0,01)$ dos demais grupos e da maior média de $4,77 \mathrm{mg} / \mathrm{dL}$ do grupo $B$ (Tabela 5; Figura 2). Traver et al. (1976) encontraram valor sérico de $P$ igual $3,6 \mathrm{mg} / \mathrm{dL}$ e $\%$ DRCr- $\mathrm{P}$ igual a $0,02 \%$ em cavalo recebendo dieta deficiente de $\mathrm{P}$. Contrastando com este valor e a semelhança do que ocorreu com o grupo B nesta pesquisa, ainda segundo os autores acima, em outro cavalo com excesso de $\mathrm{P}$ na dieta, apresentando clinicamente HPTNS o valor encontrado foi igual a 4,9 mg P sérico/dL e o \% DRCr- $P$ igual a 4\%. Traver et al. (1976) estudaram a deficiência de $\mathrm{P}$ em cavalos através da determinação do \% $\mathrm{DRCr}-\mathrm{P}$ e encontraram valor igual a $0,02 \%$. Os autores relataram também valores variando de $0 \%$ a $0,5 \%$ em cavalos supostamente normais.

No grupo $D(0,23 \% \mathrm{P})$ as potras receberam $14,04 \mathrm{~g} \mathrm{P} / \mathrm{dia}$ e os ingredientes utilizados na composição da dieta foram idênticos ao tratamento $A$ $(0,30 \% \mathrm{P}-18 \mathrm{~g} \mathrm{P} / \mathrm{dia})$, exceto que no grupo $A$ foi adicionado fosfato monosódico e continha nível normal de cálcio (Tabela 1 ). O decréscimo das médias $(P>0,05)$ de 1,25\% ( $0 \mathrm{~h})$ a 0,79\% (120 h) entre os tempos analisados do grupo $C$ podem ser expressos pela equação de regressão polinomial cúbica para tempos $\left(R^{2}=0,93\right)$ (Tabela 3$)$.

No tratamento $D$ as exigências de $P$ foram atendidas em $76,67 \%$ do NRC $(1978$ - 0,30\% P), 104,55\% do NRC (1989 - 0,22\% P) ou em $88,46 \%$ do NRC $(2007-0,26 \% P)$ para $P$ na MS base. Diferença significativa $(P<0,01)$ 
entre tempos dos tratamentos foram detectadas apenas em relação ao grupo B a partir do tempo de $48 \mathrm{~h}$ do início das dietas (Tabela 4; Figura 1).

A disponibilidade do P para pôneis de vários suplementos foi analisada por Hintz \& Schryver (1972) que constataram o efeito do fosfato dicálcico na dieta de pôneis determinando aumento excreção urinária de $11,2 \mathrm{mg} / \mathrm{kg}$ e decréscimo para $6,8 \mathrm{mg} / \mathrm{kg} \mathrm{PV} /$ dia com dieta basal (60\% de milho). Em outros estudos Hintz et al. (1973) verificaram que a digestibilidade real do P do farelo trigo foi de $29,5 \% \pm 3,0 \%$, representando metade da disponibilidade do $\mathrm{NaH}_{2} \mathrm{PO}_{4}=$ o que deve ser levado em consideração no cálculo de rações para eqüinos e Schryver et al. (1971b) consideraram que a digestibilidade real de vários ingredientes pode variar de $30 \%$ a $45 \%$ e média de $33 \%$ para $P$ fitico de cereais. Com base nas afirmações acima, analogicamente a dieta do grupo $D$ que continha como fonte $P$ exclusivamente grãos cereais podem ter comparada ao grupo $A$, com adição de $P$ inorgânico, menor proporção de excreção de $P$ na urina (Tabela 4; Figura 1).

$\mathrm{Na}$ análise conjunta dos tratamentos nos tempos, a média de 1,45\% do grupo $D$ diferiu $(P<0,01)$ significativamente da média de 9,84\% (B) para o \% DRCr- P (Tabela 5; Figura 2). A literatura revisada raramente aborda a carência de $\mathrm{P}$ na alimentação de eqüinos, talvez por este elemento representar maior problema em relação ao seu excesso na dieta. Entretanto, comportamentos semelhantes ao da presente pesquisa em relação ao metabolismo do P foram descritos em outros estudos. Schryver et al. (1971a) estudaram em pôneis a combinação de dietas com 0,40\% Ca (Ca normal) e $0,20 \% \mathrm{P}$ baixo ( $\mathrm{P}$ basal). Foi observado redução na excreção de $\mathrm{P}$ na excreção urinária. 


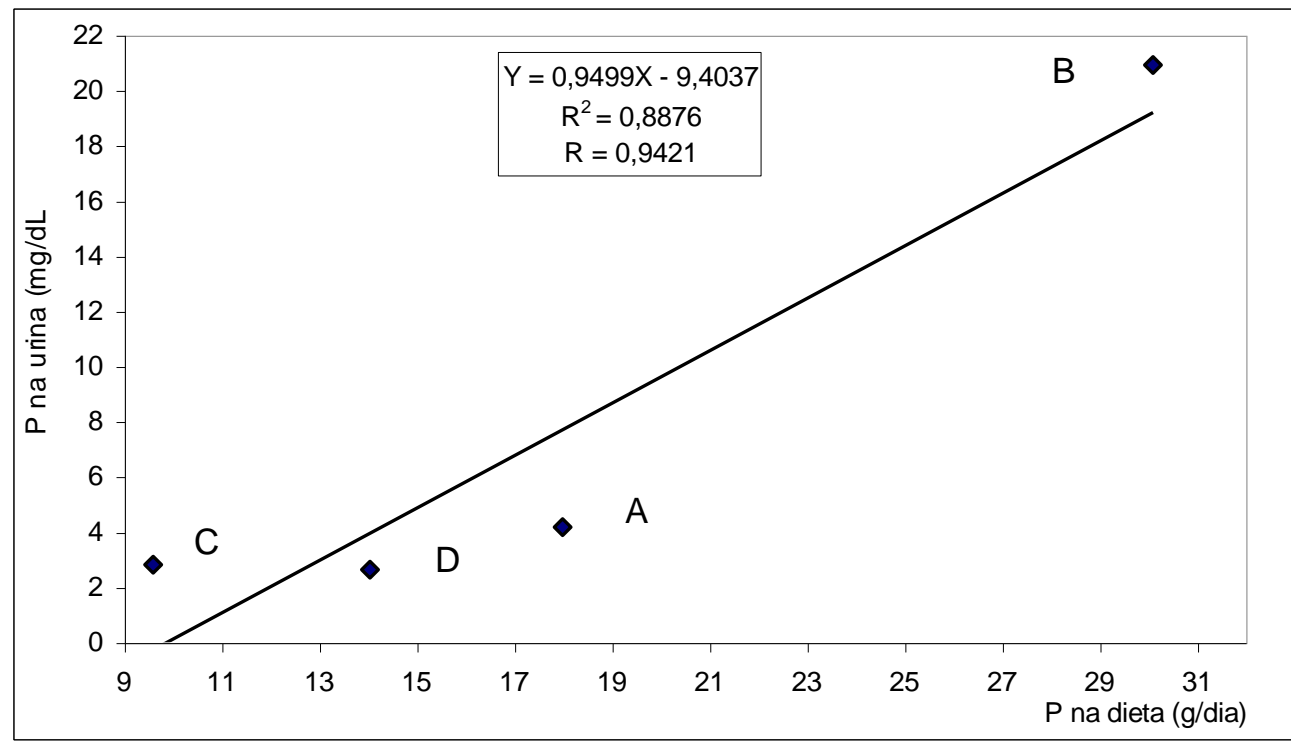

Figura 2 - Análise conjunta das médias de P na urina $(\mathrm{mg} / \mathrm{dL})$ nos tempos de 0, 48, 72, 96 e 120 horas em função dos teores de $\mathrm{P}$ na ração $(\mathrm{g} / \mathrm{kg})$ segundo os tratamentos estudados

A excreção renal de $\mathrm{P}$ foi diretamente proporcional à ingestão, ou seja, maior teor na dieta determinou proporcionalmente maior excreção urinária de P. Os resultados desta pesquisa concordam com Schryver \& Hintz (1972a) os quais assinalaram que a excreção renal foi o mecanismo mais importante na manutenção da homeostasia do $\mathrm{P}$ em pôneis, ao se constatar que a excreção fecal endógena de $P$ média de $10 \mathrm{mg} / \mathrm{kg} \mathrm{PV} / \mathrm{dia}$, não variou com a ingestão e que $\mathrm{o}$ aumento da quantidade de $\mathrm{P}$ na dieta determinou $\mathrm{O}$ aumento da quantidade absorvida. Os autores acima observaram ainda que, o excesso de $P$ na dieta interferiu com a absorção intestinal do $\mathrm{Ca}$, entretanto, com alto teor de Ca $(1,50 \%)$ não houve interferência na absorção e excreção de $P$; Joyce et al. (1971) encontraram valores variando de $116 \mathrm{mg}$ a $224 \mathrm{mg} / \mathrm{dL} P$ na urina de sete cavalos hospitalizados apresentando deformidades ósseas e HPNS. A análise da dieta revelou relação Ca:P igual a 1:10,5; Argenzio et al. (1974) estudaram em doze cavalos a homeostasia do Ca e $\mathrm{P}$ observando que nos 
animais paratireoidectomizados havia aumento significativo $(P<0,01)$ dos níveis de $\mathrm{P}$ sangüíneo de 3,66 mg no grupo controle para $4,62 \mathrm{mg} / \mathrm{dL} \mathrm{P}$ no soro nos animais do grupo paratireoidectomizados. Estas observações foram relevantes ao comprovar experimentalmente em eqüinos a função da paratireóide na regulação da homeostasia do $\mathrm{P}$ sanguíneo via excreção urinária do excesso de $P$. Neste estudo foram anotados aumentos $(P<0,05)$ de $P$ na urina de 4,15 mg (0 h) para $31,20 \mathrm{mg} / \mathrm{dL} \mathrm{P}(96 \mathrm{~h})$ no grupo B (Tabela 4; Figura 1), comprovando o mencionado pelos autores acima e por Nordin \& Peacock (1969) ao descreverem em ratos paratireoidectomizados a mesma experiência. Ainda, em concordância com os autores acima, os resultados desta pesquisa evidenciaram ativo controle dos mecanismos homeostáticos na depuração renal de P.

\section{Conclusões}

O \% DRCr- $\mathrm{P}$ é índice que detectou em tempo hábil (48 h) com probabilidade maior que $99 \%$ se a dieta de eqüinos tinha excesso de $P$. Eqüinos alimentados com excesso de $\mathrm{P}$ na ração excretaram mais $\mathrm{P}$ na urina com probabilidade maior que de $95 \%$ quando avaliados a partir de 48 horas do início na dieta.

Animais com dietas deficientes em $P$, grupos $C$ e $D$, mostraram as menores médias do \%DRCr- $\mathrm{P}$ e de excreção de $\mathrm{P}$ na urina comparados aos animais do grupo A com dieta equilibrada em $\mathrm{P}$ e $\mathrm{B}$ com excessivo $\mathrm{P}$ na dieta .

A coleta simultânea de urina e soro para determinação do \% $\mathrm{DRCr}-\mathrm{P}$ é técnica de fácil execução, praticidade, baixo custo, apresenta rapidez na execução e viabiliza emissão de resultados para tomada de decisão a tempo, ou seja, antes que as osteopatias subclínicas determinem quadros clínicos irreversíveis ao esqueleto dos eqüinos. 
Carvalho, P.R., d'Arce, R.D., Machado Neto, R. et al. Aplicação da técnica da taxa de depuração renal de creatinina para avaliação do "status" do fósforo em eqüinos. PUBVET, Londrina, V. 2, N. 45, Art\#441, Nov2, 2008.

\section{Referências Bibliográficas}

ARGENZIO, R.A.; LOWE, J.E.; HINTZ, H.F. Calcium and phosphorus homeostasia in horses. Journal of Nutrition, v.104, n.1, p.18-27, 1974.

ASSOCIATION OF OFFICIAL ANALYTICAL CHEMISTS - AOAC. Official methods of analysis. 11.ed. Washington: AOAC International, 1970. 1015p.

BOURKE, J.M. Feeding of thoroughbred horses. Australian Veterinary Journal, v.44, n.5, p. 241-245, 1968.

CAPLE, I.W.; DOAKE, P.A.; ELLIS, P.G. Assessment of the calcium and phosphorus nutrition in horses by analysis of urine. Australian Veterinary Journal, v.58, n.4, p. 125-131, 1982a.

CAPLE, I.W.; BOURNE, J.M.; ELLIS, P.G. An examination of the calcium and phosphorus nutrition of throughbred race horses. Australian Veterinary Journal, v. 58, n.4, p.132$135,1982 b$.

COFFMAN, J. Percent creatinine clearance ratios. Veterinary Medicine Small and Animal Clinician, v.75, n.4, p.671-676, 1980.

CUDDEFORD, D.; WOODHEAD, A.; MUIRHEAD, R. Potencial of alfalfa as a source of calcium for calcium deficient horses. Veterinary Record, v.126, n.17, p.425-429, 1990.

CYMBALUK, N.F.; CHRISTENSEN, D.A. Nutrient utilization of pelleted and unpelleted forages by ponies. Canadian Journal of Animal Science, Ottawa, v.66, n.2, p.237-244, 1986.

EDWARS, D.J.; BROWNLOW, M.A.; HUTCHINS, D.R. Indices of renal function: reference values in normal horses. Australian Veterinary Journal, v.66, n.2, p.60-63, 1989.

GANS, J.H.; MERCER, P.F. In: SWENSON, M.J. (Ed.). Duke's Physiology of domestic animals. The kidneys. Cornell University Press Ltd., London, Tenth edition, 1984. p. 507-536.

HARRIS, P.; COLLES, C. The use of creatinine clearance ratios in the prevention of equine rhabdomyolysis: a report of four cases. Equine Veterinary Journal, v.20, n.6, p.459463, 1988.

HINTZ, H.F.; SCHRYVER, H.F. Availability to ponies of calcium and phosphorus from various supplements. Journal of Animal Science, v.34, n.6, p. 979-980, 1972.

HINTZ, H.F.; WILLIAMS, A.J.; ROGOFF, J. et al. Availability of phosphorus in wheat bran when fed to ponies. Journal of Animal Science, v.36, n.3, p.522-525, 1973.

HINTZ, H.F.; SCHRYVER, H.F. Nutrition and bone development in horses. Journal of the American Veterinary Medical Association, v.168, n.1, p. 39-44, 1976.

HINTZ, H.F.; HINTZ, R.L.; VAN VLECK, L.D. Growth rate of thoroughbreds, effect of age of dam, year and month of birth, and sex of foal. Journal of Animal Science, v.48, n.3, p.480-487, 1979.

JOYCE, J.R.; PIERCE, K.R.; ROMANE, W.M. et al. Clinical study of nutritional secondary hyperparathyroidism in horses. Journal of the American Veterinary Medical Association, v.158, p.2033-42, 1971.

KNIGHT, D.A.; GABEL, A.A.; REED, S.M. et al. Correlation of dietary mineral to incidence and severity of metabolic bone disease in Ohio and Kentuck. In: Annual Convention of the American Association Equine Practit, 31. KANSAS, 1985. Anais... Lexington, Ohio State University, 1985 , p. 445-461.

KNUDSEN, E. Renal clearance studies on the horse. I: inulin, endogenous creatinine and urea. Acta Veterinaria Scandivica, v.1, p.52-66, 1959.

KOHN, C. W.; STRASSER, S.L. 24-hour renal clearance and excretion of endogenous substances in the mare. American Journal of Veterinary Research, v.47, n.6, p.13321337, 1986.

KROOK, L. Dietarary calcium-phosphorus and lameness in the horse. Cornell Veterinarian, v.58, p.58-73, 1968. Supplement.

KROOK, L.; LOWE J.E. Nutritional secondary hyperparathyroidism in the horse. Pathology Veterinarian, v.1, p.1-98, 1964. Supplement 1. 
Carvalho, P.R., d'Arce, R.D., Machado Neto, R. et al. Aplicação da técnica da taxa de depuração renal de creatinina para avaliação do "status" do fósforo em eqüinos. PUBVET, Londrina, V. 2, N. 45, Art\#441, Nov2, 2008.

LANE, V.M.; MERRITT, A.M. Reliability of single-sample phosphorus fractional excretion determination as a measure of daily phosphorus renal clearance in equids. American Journal of Veterinary Research, v.44, n.3, p. 500-502, 1983.

LOPES, J.B.; FURTADO, C.E.; VITTI, D.M.S.S. et al. Metabolismo do fósforo em eqüinos. 2. Efeitos de diferentes níveis de fósforo dietético. Revista Brasileira de Zootecnia, v.32, n.6, p.1348-1353, 2003.

MASON, D.K.; WATKINS, K.L.; MCNIE, J.T. Diagnosis, treatment and prevention of nutritional secondary hyperparathyroidism in throughbred race horses in Hong kong. Equine Practice, v.10, n.3, p.10-17, 1988.

NATIONAL RESAEARCH COUNCIL - NRC. Nutrient requeriments of domestic animals, 6. Nutrient requeriments of horses. Washington, D.C.: National Academy of Sciences, 1978. 33 p.

NATIONAL RESEARCH COUNCIL - NRC. Nutrient requirements of horses. 5.ed. Washington, D.C.: National Academy of Sciences, 1989. 100p.

NATIONAL RESAEARCH COUNCIL - NRC. Nutrient requeriments of horses. 6th rev. ed. Washington, D.C.: National Academy of Sciences, 2007. 341 p.

NORDIN, B.E.; PEACOCK, M. Role of kidney in regulation of plasma calcium. Lancet, v.13, n.2, p.1280-1283, 1969.

NORDIN, B.E. Primary and secondary hyperparathyroidism. Advances Internal Medicine, v.9, p.81-105, 1958.

RAWLINGS, C.A.; BISGARD, G.E. Renal clearance and excretion of endogenous substances in the small pony. American Journal of Veterinary, v.36, n.1, p.45-48, 1975.

SAS Institute. SAS / STAT. User's Guide: statistical version. Cary, NC: SAS Institute, 1994.

SCHRYVER, H.F.; CRAIG, P.H.; HINTZ, H.F. Calcium metabolism in ponies fed varying levels of calcium. Journal of Nutrition, v.100, n.8, p.955-964, 1970.

SCHRYVER, H.F.; HINTZ, H.F.; CRAIG, P.H. Calcium metabolism in ponies fed high phosphorus diet. Journal of Nutrition, v.101, n.2, p.259-264, 1971a.

SCHRYVER, H.F.; HINTZ, H.F.; CRAIG, P.H. Phosphorus metabolism in ponies fed varying levels of phosphorus. Journal of Nutrition, v.101, n.9, p. 1257-1264, 1971b.

SCHRYVER, H. F.; HINTZ, H.F. Calcium and phosphorus requeriments of the horse: A review. Feedstuffs, v.44, p.35-36, 38, 1972a.

SCHRYVER, H.F.; HINTZ, H.F.; CRAIG, P.H. et al. Site of phosphorus absorption from the intestine of the horse. Journal of Nutrition, v.102, n.1, p.143-148, 1972b.

SCHRYVER, H.F.; HINTZ, H.F.; LOWE, J.E. et al. Mineral composition of young horses. Journal of Nutrition, v.104, n.1, p.124-132, 1974.

TEETER, S.M.; STILLIONS, M.C.; NELSON, W.E. Maintenance levels of calcium and phosphorus in horses. Journal of the American Veterinary Medical Association Journal, v.151, n.12, p.1625-1628, 1967.

TRAVER, D.S.; COFFMAN, J.R.; MOORE, J.N. et al. Urine clearance ratios as diagnostic acid in equine metabolic disease. Proceedings of the American Association of Equine Practitioners... St. Louis, v.22, p.177-183, 1976.

TRAVER, D.S.; SALEN, C.; COFFMAN, J.R. et al. Renal metabolism of endogenous substances in the horse: volumetric vs clearance ratio methods. Journal of Equine Medicine and Surgery, v.1, n.11, p. 378-382, 1977.

VAN DOORN, D.A.; EVERTS, H.; WOUTERSE, H.; BEYNEN, A.C. The apparent digestibility of phytate phosphorus and the influence of supplemental phytase in horses. Journal of Animal Science, v.82, n.6, p.1756-1763, 2004.

WHITLOCK, R.H.; SCHRYVER, H.F.; KROOK, L.; HINTZ, H.F.; CRAIG, P.H. The effects of high dietary calcium in horses. In: American Association of Equine Practitioners, 16 th. Anais... p.127-134, 1971.

ZATSMAN, M.L.; CLARKE, L.; RAY, W.F. et al. Renal function of the pony and horse. American Journal of Veterinary Research, v.43, n.4, p.608-612, 1982. 
Carvalho, P.R., d'Arce, R.D., Machado Neto, R. et al. Aplicação da técnica da taxa de depuração renal de creatinina para avaliação do "status" do fósforo em eqüinos. PUBVET, Londrina, V. 2, N. 45, Art\#441, Nov2, 2008.

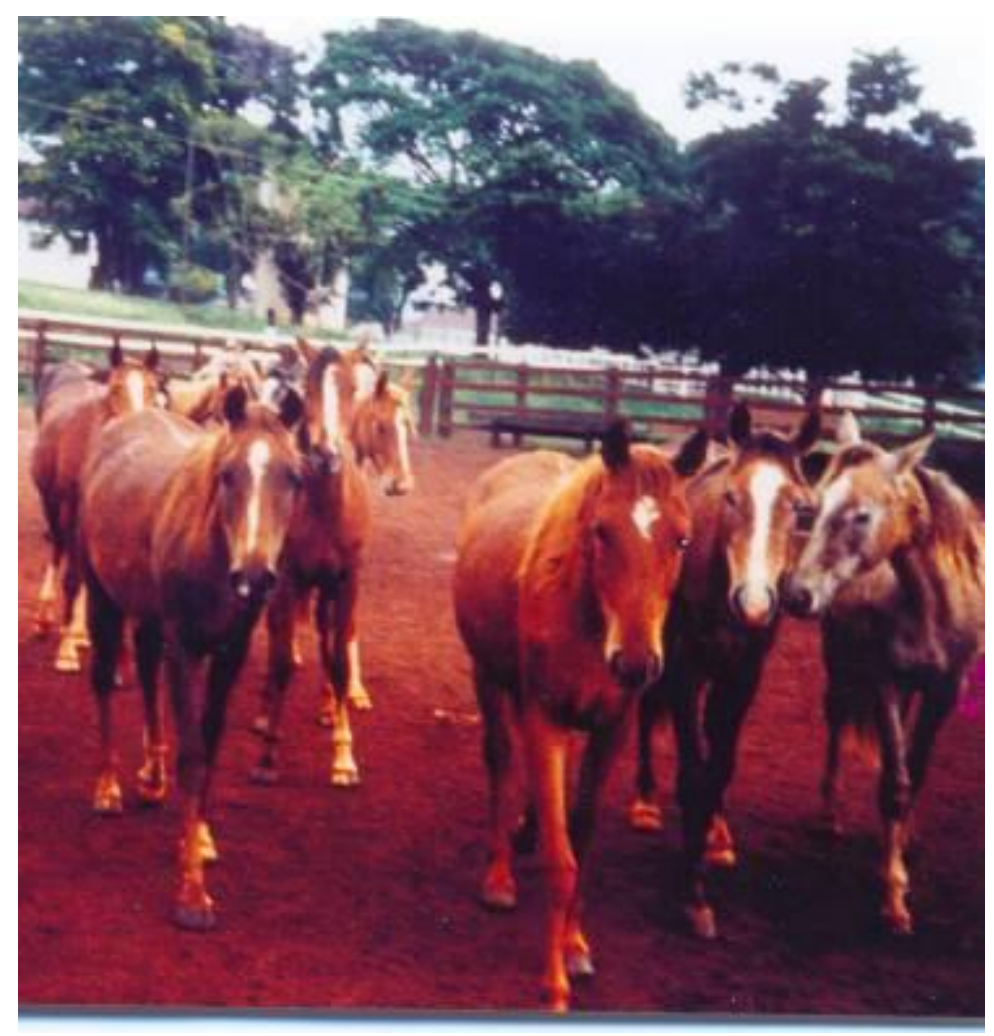

Figura - Potras mestiço árabe e árabe utilizadas no experimento 